\title{
On the differential equation for the conventional tide-well system
}

\section{B.J. Noye}

This paper discusses solutions to the differential equation

$$
d Y / d \tau+\beta^{-1}|Y|^{\frac{3}{2}} \operatorname{sgn}(Y)=d X / d \tau
$$

which governs the height

$$
Z(\tau)=X(\tau)-Y(\tau)
$$

of the non-dimensional water level inside a conventional

tide-well when the corresponding height of the sea-level outside is $X(\tau)$. A perturbation solution correct to $O\left(B^{4}\right)$, for small $B$, for the particular case $X=\sin t$ described previously has been extended to $O\left(\beta^{6}\right)$ for any function $X(\tau)$ differentiable at least three times. Two special cases, where $X(\tau)$ is a single sinusoid and the sum of two sinusoids, are treated in detail.

\section{Introduction}

In [3] a class of differential equations which governed the response of three types of tide-well systems was discussed. In this paper, the non-linear response of the conventional type of tide-well, in which the water inside the well is connected to that outside by means of a circular orifice near the bottom of the well, is further investigated. It was shown in [2] that the differential equation governing the response of the conventional tide-well system to the fluctuating sea-level $h_{0}(t)$ is

Received 5 May 1972. 


$$
d H / d t+C|H|^{\frac{1}{2}} \operatorname{sgn}(H)=d h_{0} / d t,
$$

where $h_{\omega}(t)$ is the level inside the well, $t$ is the time, and the excess external head is

$$
H(t)=h_{0}(t)-h_{w}(t)
$$

The tide-well parameter is

$$
C=C_{c}(2 g)^{\frac{1}{2}} A_{p} / A_{w},
$$

where $C_{c}$ is the coefficient of contraction of the orifice, $g$ is the gravitational constant, $A_{p}$ is the area of cross-section of the orifice, and $A_{w}$ is the area of cross-section of the well. We are interested in finding the well response

$$
h_{w}=h_{0}-H
$$

for a given input $h_{0}(t)$.

A non-dimensional form of the tide-well equation is obtained by rewriting it in terms of the dimensionless input $X=h_{0} / a$, the dimensionless head response $Y=H / \alpha$ and the dimensionless frequency $\beta=\omega \sqrt{a} / C$, where $X$ and $Y$ are functions of $\tau=\omega t$, and $a$ and $\omega$, respectively, are the characteristic amplitude and circular frequency of the sea-level oscillations. Equation (1.1) then becomes

$$
\beta(d Y / d \tau)+|Y|^{\frac{1}{2}} \operatorname{sgn}(Y)=\beta(d X / d \tau)
$$

The head response $Y$ for a given input $X$ must be found from this equation; the dimensionless well response, $z=h_{w} / a$, is then given by

$$
Z=X-Y .
$$

In [3] much was learned about the response of this system to an arbitrary input from the steady state response to a sinusoidal input of fixed amplitude $a$ and circular frequency $\omega$, namely,

$$
h_{0}=a \sin \omega t \text {, }
$$


that is, $x=\sin \tau$.

Using a perturbation solution correct to $O\left(\beta^{4}\right)$, for small $\beta$, the output was found to be

$$
z=\sin \tau-\beta^{2} \cos ^{2} \tau \operatorname{sgn}(\cos \tau)-4 \beta^{4} \cos ^{2} \tau \sin \tau+O\left(\beta^{6}\right) .
$$

Writing the coefficients of $\beta^{2}$ and $\beta^{4}$ in their Fourier series, namely,

$$
\begin{aligned}
\cos ^{2} \tau \operatorname{sgn}(\cos \tau) & =\sum_{m=1}^{\infty} C_{m} \cos (2 m-1) \tau, \\
\cos ^{2} \tau \sin \tau & =\sum_{m=1}^{\infty} K_{m} \sin (2 m-1) \tau,
\end{aligned}
$$

gave

(1.9) $z=\alpha_{f}^{(1)} \sin \left(\tau-\theta_{f}^{(1)}\right)-\beta^{2} \sum_{m=2}^{\infty}\left(c_{m}^{2}+\beta^{4} K_{m}^{2}\right)^{\frac{1}{2}} \sin \left\{(2 m-1) \tau-\phi_{m}\right\}+O\left(\beta^{6}\right)$, where

$$
\alpha_{f}^{(1)}=1-\left(K_{1}-\frac{1}{2} C_{1}^{2}\right) \beta^{4}+O\left(\beta^{6}\right)
$$

and

$$
\theta_{f}^{(1)}=C_{1} \beta^{2}+O\left(\beta^{6}\right)
$$

With

$$
c_{m}=\frac{8(-1)^{m}}{\pi(2 m-3)(2 m-1)(2 m+1)}, m=1,2,3, \ldots
$$

and

$$
\begin{aligned}
K_{m} & =1 \text { for } m=1,2, \\
& =0 \text { otherwise, }
\end{aligned}
$$

then the amplitude response of the oscillation in the output with the same frequency as the input is, to $O\left(\beta^{4}\right)$,

$$
\alpha_{f}^{(1)}=1-0.64 \beta^{4}
$$

with corresponding phase-lag 


$$
\theta_{f}^{(1)}=0.85 \beta^{2}
$$

Therefore, for a pure sinusoidal fluctuation of sea level, the water level inside a conventional tide-well consists of a fundamental oscillation at the same frequency as the input, but of reduced amplitude and with a phase-lag, plus odd harmonics of rapidy decreasing amplitude.

Subsequently, Brown [1] examined (1.5) with (1.7) from a different point of view. By meáns of a change of variable he transformed (1.5) to the equation of motion for a pendulum with viscous damping, one of the standard examples of a non-linear differential equation. He discussed the behaviour of the solutions of the transformed equation as represented in the phase plane and showed how they corresponded to the perturbation solution of Noye [3].

In the following a perturbation solution for (1.5) is obtained correct to $O\left(\beta^{6}\right)$ for any input $X(\tau)$ which is differentiable at least three times. The result is then used to estimate the response of the system to a pure sinusoidal input to a higher order of accuracy than before, and to an input consisting of the sum of two sinusoids of differing amplitude and frequency.

Because (1.1) and (1.5) are non-linear the principle of superposition of solutions does not hold, so no unique response function can be defined which relates the output of the system to an input which consists of the sum of a number of different waves. For an input consisting of the sum of two sinusoidal waves, it is shown that odd harmonics of the two fundamental frequencies occur in the output, together with contributions at frequencies which are the sum and difference of various multiples of the fundamental frequencies. The implication on the interpretation to be placed on the results of analysis of tide-well records is profound. Harmonics of the oscillations in the sea-level together with linear combinations of their frequencies will appear in the tide-well record. Therefore one cannot be sure whether small peaks which appear in the Fourier spectrum of a tidewell record are contributions occurring in the sea-level oscillation or merely due to the non-linear effects of the orifice. 


\section{Asymptotic solution for small $B$}

Successive approximations to the steady-state solution of (1.5) can be found, for small $B$ and an arbitrary input $X$, by the method described in [3]. Let $y=\sum_{n=1}^{\infty} y_{n}$, where $y_{n+1}=o\left(y_{n}\right)$ compared with $\beta$. Successive approximations

$$
y^{(r)}=\sum_{n=1}^{r} y_{n}, r=1,2, \ldots,
$$

are found where $y^{(r)} \rightarrow Y$ as $r \rightarrow \infty$. Substitution in (1.5) gives (2.1) $B d / d \tau\left(y_{1}+y_{2}+\ldots\right)+\left|y_{1}+y_{2}+\ldots\right|^{\frac{1}{2}} \operatorname{sgn}\left(y_{1}+y_{2}+\ldots\right)=\beta d X / d \tau$. Letting $\beta \rightarrow 0$, one finds to leading order in $\beta$,

$$
\left|y_{1}\right|^{\frac{3}{2}} \operatorname{sgn}\left(y_{1}\right)=B d X / d \tau
$$

with

$$
\operatorname{sgn}\left(y_{1}\right)=\operatorname{sgn}(d X / d \tau),
$$

whence

$$
Y_{1}=\beta^{2}(d X / d \tau)^{2} \operatorname{sgn}(d X / d \tau)
$$

The quantity $Y_{2}$ can now be obtained by substituting this value of $Y_{1}$ into a rearranged form of (2.1), namely,

$$
\left|y_{1}+y_{2}+\ldots\right|=B^{2}\left\{d / d \tau\left(X-y_{1}-y_{2}-\ldots\right)\right\}^{2}
$$

with

$$
\operatorname{sgn}\left(y_{1}+y_{2}+\ldots\right)=\operatorname{sgn}\left\{d / d \tau\left(X-y_{1}-y_{2}-\ldots\right)\right\} .
$$

Taking again the limit $\beta \rightarrow 0$ one finds that $y_{2}=O\left(\beta^{4}\right)$ and

$$
y^{(2)}=\beta^{2}\left(\frac{d X}{d \tau}\right)^{2} \operatorname{sgn}\left(\frac{d X}{d \tau}\right)-4 \beta^{4}\left(\frac{d X}{d \tau}\right)^{2}\left(\frac{d^{2} X}{d \tau^{2}}\right) \text {. }
$$

The third approximation $y^{(3)}$ is found by substituting (2.6) into (2.4) 
and (2.5) and taking the limit $\beta \rightarrow 0$, which gives

(2.7) $y^{(3)}=\beta^{2}\left(\frac{d X}{d \tau}\right)^{2} \operatorname{sgn}\left(\frac{d X}{d \tau}\right)-4 \beta^{4}\left(\frac{d X}{d \tau}\right)^{2}\left(\frac{d^{2} X}{d \tau^{2}}\right)$

$$
+4 B^{6}\left\{5\left(\frac{d X}{d \tau}\right)^{2}\left(\frac{d^{2} X}{d \tau^{2}}\right)^{2}+2\left(\frac{d X}{d \tau}\right)^{3}\left(\frac{d^{3} X}{d \tau^{3}}\right)\right\} \operatorname{sgn}\left(\frac{d X}{d \tau}\right) \text {. }
$$

Working with the asymptotic solution correct to $O\left(\beta^{6}\right)$, the tide-well output becomes now

$$
\text { (2.8) } \begin{aligned}
z=X-\beta^{2}\left(\frac{d X}{d \tau}\right)^{2} \operatorname{sgn}\left(\frac{d X}{d \tau}\right) & +4 \beta^{4}\left(\frac{d X}{d \tau}\right)^{2}\left(\frac{d^{2} X}{d \tau^{2}}\right) \\
& -4 \beta^{6}\left(\frac{d X}{d \tau}\right)^{2} \operatorname{sgn}\left(\frac{d X}{d \tau}\right)\left\{5\left(\frac{d^{2} X}{d \tau^{2}}\right)^{2}+2\left(\frac{d X}{d \tau}\right)\left(\frac{d^{3} X}{d \tau^{3}}\right)\right\}+o\left(\beta^{8}\right) .
\end{aligned}
$$

Consider now two separate inputs: a single sinusoid, and a sum of two sinusoids with different frequencies.

\section{Single sinusoidal input}

With the input $X=\sin \tau$, the tide-well output is

(3.1) $z=\sin \tau-\beta^{2} \cos ^{2} \tau \operatorname{sgn}(\cos \tau)-4 \beta^{4} \cos ^{2} \tau \sin \tau$

$$
-4 \beta^{6} \cos ^{2} \tau \operatorname{sgn}(\cos \tau)\left\{5-7 \cos ^{2} \tau\right\}+O\left(B^{8}\right) \text {. }
$$

Expanding the terms in $\beta^{2}, \beta^{4}$ and $\beta^{6}$ in Fourier series yields

$$
\begin{aligned}
Z=\sin \tau-\beta^{2}\left\{\sum_{m=1}^{\infty} C_{m} \cos (2 m-1) \tau\right\}-\beta^{4} & \left\{\sum_{m=1}^{\infty} K_{m} \sin (2 m-1) \tau\right\} \\
& -\beta^{6}\left\{\sum_{m=1}^{\infty} D_{m} \cos (2 m-1) \tau\right\}+O\left(\beta^{8}\right),
\end{aligned}
$$

where $C_{m}, K_{m}$ are given by $(1.10),(1.11)$ and

$$
D_{m}=6 C_{m}-7 C_{m-1} \text { with } C_{0}=2
$$

Rearranging (3.2) the tide-well response takes the form 
(3.3) $z=\left\{\left(1-\beta^{4}\right) \sin \tau-\left(\frac{8 \beta^{2}}{3 \pi}+2\left(\frac{8}{\pi}-7\right) \beta^{6}\right) \cos \tau\right\}$

$$
\begin{aligned}
& -\beta^{2}\left\{\beta^{2} \sin 3 \tau+\frac{8}{15 \pi}\left(1-29 \beta^{4}\right) \cos 3 \tau\right\} \\
& -\sum_{m=3}^{\infty}\left(C_{m} \beta^{2}+D{ }_{m} \beta^{4}\right) \cos (2 m-1) \tau+O\left(\beta^{8}\right) .
\end{aligned}
$$

The contribution to $z$ at the fundamental frequency can be written in the form

$$
z_{f}=\alpha_{f}^{(2)} \sin \left(\tau-\theta_{f}^{(2)}\right)
$$

where

$$
\begin{gathered}
\alpha_{f}^{(2)}=1-\left(1-\frac{32}{9 \pi^{2}}\right) \beta^{4}+O\left(B^{8}\right), \\
\theta_{f}^{(2)}=\frac{8 \beta^{2}}{3 \pi}-\left(14-\frac{56}{3 \pi}+\frac{512}{\pi^{3}}\right) \beta^{6}+O\left(\beta^{8}\right),
\end{gathered}
$$

that is, $\alpha_{f}^{(2)}$ and $\theta_{f}^{(2)}$ are the amplitude response and the phase lag of the output at the fundamental frequency, correct to $O\left(\beta^{6}\right)$.

To $O\left(\beta^{4}\right)$ these are the same as $\alpha_{f}^{(1)}$ and $\theta_{f}^{(1)}$ given by $(1.12)$ and $(1.13)$

The ratio of the amplitude of the third harmonic to the amplitude of the fundamental in the output is a measure of the distortion of the output. By (3.3) the amplitude of this harmonic is

$$
\alpha^{*}=\frac{8 \beta^{2}}{15 \pi}\left\{I+\left(\frac{225 \pi^{2}}{128}-29\right) \beta^{4}\right\}+o\left(\beta^{8}\right),
$$

and the required ratio is

$$
\frac{\alpha^{*}}{\alpha_{f}^{(2)}}=\frac{8 \beta^{2}}{15 \pi}\left\{1+\left(\frac{225 \pi^{2}}{128}+\frac{32}{9 \pi^{2}}-30\right) \beta^{4}\right\}+O\left(\beta^{8}\right) .
$$

These results show the presence of higher harmonics in the response of a conventional tide-well to a sinusoidal input. Therefore care must be taken in the interpretation of the results of Fourier analysis of tide 
records. Some of the components found may not exist in the fluctuations of the sea level outside the well; they may appear in the oscillations of the water inside the well simply because of the non-linearity of the tide-well with an orifice.

If the input $h_{0}$ is a sum of sinusoidal functions with varying frequencies, namely,

$$
h_{0}=\sum_{n=1}^{N} a_{n} \sin \left(\omega_{n} t+\theta_{n}\right) \text {, }
$$

the position becomes more complicated. The non-linearity of the system does not permit the superposition of separate sol.utions for inputs

$$
h_{0}^{(n)}=a_{n} \sin \left(\omega_{n} t+\theta_{n}\right) .
$$

One would expect the output of such systems to contain components with sums and differences of frequencies

$$
\omega_{i} \pm \omega_{j}, i, j=1,2,3, \ldots, N, i \neq j,
$$

as well as the fundamental frequencies

$$
\omega_{n}, n=1,2,3, \ldots, N \text {, }
$$

and their harmonics

$$
p \omega_{n}, p, n=1,2,3, \ldots .
$$

The following questions can be asked: Does the tide-well, because of its non-linear characteristics, give measurable oscillations in the tide record which do not exist in the sea-level fluctuations outside the well? Is it possible that under certain circumstances these spurious oscillations contribute to, or even are mistaken for small constituents such as certain shallow water tidal components?

As a test case, we examine the response to an input which consists of the sum of two sinusoids of slightly differing frequencies.

\section{Sum of two sinusoids as input}

Let the sea-level fluctuation take the form 
$(4.1)$

$$
h_{0}=a \sin \omega t-a n(n+1)^{-1} \sin (1+1 / n) \omega t .
$$

The dimensionless input in (1.5) is now

$$
X=\sin \tau-n(n+1)^{-1} \sin (1+1 / n) \tau .
$$

The solution (2.8) applies for small values of $\beta=\omega \sqrt{a} / C$, whence

$$
z=X-\beta^{2} w_{1}+4 \beta^{4} w_{2}+o\left(\beta^{6}\right),
$$

where, with $\tau=n \theta$,

$$
\text { (4.4) } \begin{aligned}
W_{1} & =(d X / d \tau)^{2} \operatorname{sgn}(d X / d \tau) \\
& =\left\{1+\frac{1}{2} \cos (2 n \theta)+\frac{1}{2} \cos (2 n+2) \theta-\cos (2 n+1) \theta-\cos \theta\right\} \cdot \\
& \cdot \operatorname{sgn}\{\sin [(2 n+1) \theta / 2] \sin (\theta / 2)\},
\end{aligned}
$$

and

$$
\begin{aligned}
W_{2} & =(d x / d \tau)^{2} \cdot\left(d^{2} x / d \tau^{2}\right) \\
& =\sum_{p=1}^{\infty} b_{p} \sin (p \theta),
\end{aligned}
$$

with

$$
\begin{gathered}
b_{p}=(-1)^{p-n+1}{ }_{r_{p} p / n}, \\
r_{p}= \begin{cases}1 / 4 & \text { for } p=n-1, n+2,3 n+1,3 n+2, \\
3 / 4 & \text { for } p=n, n+1, \\
1 / 12 & \text { for } p=3 n, 3 n+3, \\
0 & \text { otherwise. }\end{cases}
\end{gathered}
$$

Next, the Fourier series representation of $W_{1}$ is required to make possible the comparison between the output $Z$ and the input $X$, at the frequencies of the sinusoidal components of $X$ and various linear combinations of these. Since $W_{1}$ with period $2 \pi$ is an even function of $\theta$, it can be written in the form

$$
w_{1}=\frac{3}{2} a_{0}+\sum_{p=1}^{\infty} a_{p} \cos (p \theta),
$$

where 


$$
a_{p}=\frac{1}{2 \pi} \int_{0}^{2 \pi} W_{1}(\theta) \cos (p \theta) d \theta
$$

A straightforward but tedious analysis, outlined in the Appendix, yields very complicated but exact formulae for the coefficients $a_{p}$, which were used in the computations leading to Table 1 . Substitution from (4.2), $(4.5),(4.6)$ into $(4.3)$ gives, to $O\left(\beta^{6}\right)$,

$$
z=-\frac{1}{2} \beta^{2} a_{0}+\sum_{p=1}^{\infty}\left\{\left(-\beta^{2} a_{p}\right) \cos p \theta+\left(4 \beta^{4} b_{p}+c_{p}\right) \sin p \theta\right\},
$$

where

$$
c_{p}= \begin{cases}1 & \text { if } p=n, \\ -n(n+1)^{-1} & \text { if } p=n+1, \\ 0 & \text { otherwise. }\end{cases}
$$

This may be rewritten in the form

$$
z=-\frac{1}{2} B^{2} a_{0}+\sum_{p=1}^{\infty} \alpha_{p} \sin \left(p \tau / n-\varepsilon_{p}\right)
$$

where

$$
\begin{gathered}
\alpha_{p}=\left\{\beta^{4} a_{p}^{2}+\left(4 \beta^{4} b_{p}+c\right)_{p}\right\}^{\frac{1}{2}}, \\
\varepsilon_{p}=\arctan \left\{\beta^{2} a_{p} /\left(4 \beta^{4} b_{p}+c\right)\right\} .
\end{gathered}
$$

Particular interest attaches to the amplitudes of the oscillations in the well which occur at the same frequencies as the oscillations in the input, namely,

$$
\begin{gathered}
\alpha_{n}=1-\left(3-a_{n}^{2} / 2\right) \beta^{4}+o\left(\beta^{8}\right) \\
\alpha_{n+1}=n(n+1)^{-1}\left\{1-\left(3-a_{n+1}^{2} / 2\right) \beta^{4}\right\}+o\left(\beta^{8}\right) .
\end{gathered}
$$

Table 1 lists a typical set of contributions to the well response at different frequencies for a double sinusoidal input of this kind, for $\beta=0.4$ and $n=3,4, \ldots, 8$. Here, $\alpha_{1}$ gives the contribution at longer periods than the incident waves; for example, for $n=3$, the 


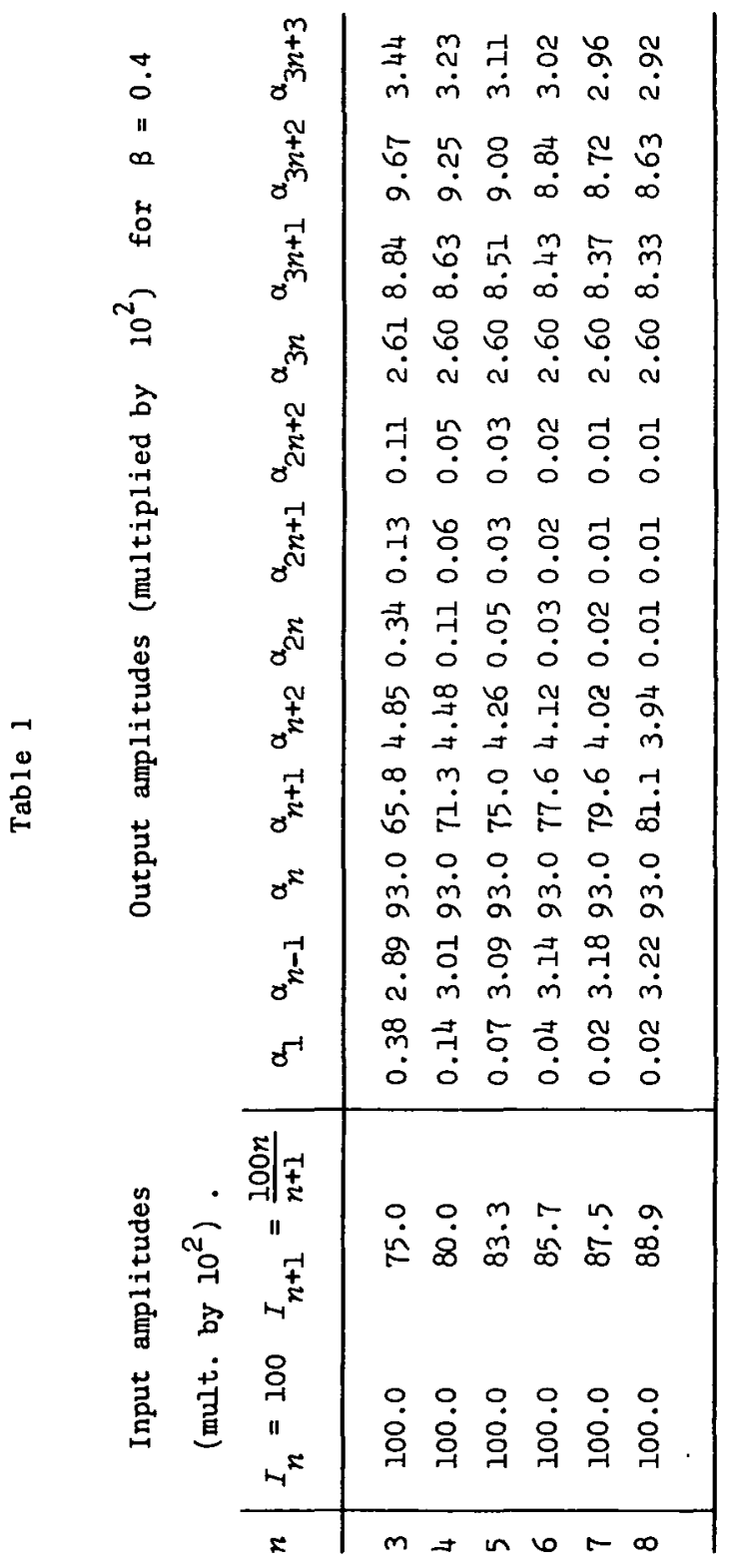


frequencies of the incident oscillations are $\omega$ and $4 \omega / 3$, and at the difference frequency $\omega / 3$ an amplitude of $0.38 \%$ of the amplitude at the frequency $\omega$ occurs.

There are also significant contributions at side-bands to the frequencies of the incident oscillations. For instance, with $n=3$, an amplitude of $2.89 \%$ of the incident amplitude at frequency $\omega$ occurs at the frequency $2 \omega / 3$ and an amplitude $6.48 \%$ of the incident amplitude at frequency $4 \omega / 3$ at the frequency $5 \omega / 3$.

The contributions to the output at harmonics of double the incident frequencies and the sum of these frequencies (see $\alpha_{2 n}, \alpha_{2 n+1}$ and $\alpha_{2 n+2}$ ) are small compared with the contributions around three-times the incident frequencies. The third harmonic of the incident frequencies are given by $\alpha_{3 n}$ and $\alpha_{3 n+3}$. For $n=3$, the third harmonic of the wave with frequency $\omega$ has an amplitude of $2.61 \%$ of the incident amplitude at that frequency, and the third harmonics of the incident frequencies are given by amplitude of $4.59 \%$ of the corresponding incident amplitude. The most striking feature of all is the large contribution at frequencies given by doubling one incident frequency and adding the other; for all values of $n$ in Table 1, $\alpha_{3 n+1}$ and $\alpha_{3 n+2}$ appear to be between 8 and $10 \%$ of the incident amplitudes. In the past, contributions at such linear combinations of the frequencies of the incident waves have been attributed to effects outside the tide-well system, for example, non-linear effects on the tides due to shallow water. Such harmonics may be wholly or partly due to the non-linear effects of the orifice. One would suspect that many of the 114 tidal constituents computed from tide-well records for Anchorage, Alaska [5], and for Southend on the Thames Estuary [4], might include non-linear contributions from the orifice. In the latter analysis 21 of the constituents have an amplitude less than 0.01 feet compared with the $M 2$ component of amplitude 6.666 feet.

\section{Conclusion}

The implications surrounding the findings of this paper are farreaching when applied to the interpretation of tide records obtained from conventional tide-wells. 
The conventional tide-well, consisting of a well with an orifice, has been shown to be a non-linear device with all the inherent disadvantages of non-linear systems. On the basis of the analysis, the water level in the well may oscillate at the frequencies of the oscillations in the sea-level, on which higher harmonics and oscillations with frequencies which are sums and differences of these frequencies are superimposed. Furthermore, the amplitudes of the resulting well oscillations are non-linear functions of the amplitudes of the sea-level oscillations, a fact which creates many difficulties in the use of tide-well records, for example, in the analysis of harbour oscillations. Pseudo-response functions give a rough idea of the attenuation and lag of a system, but they are difficult to use because the response depends on the amplitude as well as the frequency.

Problems also arise when records from a conventional tide-well system are analysed for tidal components. With an input which consists of waves with different frequencies, such as tidal components, it has been shown that the non-linearity of a system may contribute to the amplitudes of harmonics of these components and to oscillations which occur at sum and difference frequencies of these components. It is likely that some of the energy attributed to shallow water components may not, in fact, be due to influences from outside the well but rather arise from non-linear effects at the orifice.

Appendix: Computation of the Fourier coefficients

$$
a_{p}=\frac{1}{2 \pi} \int_{0}^{2 \pi} w_{1}(\theta) \cos (p \theta) d \theta
$$

In the range $(r-1) \theta_{n}<\theta<r \theta_{n}, r=1,2, \ldots,(2 n+1)$, where $\theta_{n}=2 \pi /(2 n+1)$,

$$
\operatorname{sgn}\left\{\sin \left(\frac{2 n+1}{2} \theta\right) \sin \left(\frac{\theta}{2}\right)\right\}=(-1)^{r-1} \text {. }
$$

Substituting (A.1) into (4.4) we find

$$
a_{p}=\frac{1}{2 \pi} \sum_{r=1}^{2 n+1}(-1)^{r-1} \int_{(r-1) \theta_{n}}^{r \theta} \kappa(p, \theta) d \theta,
$$

where 
(A.3) $k(p, \theta)=\cos p \theta+\frac{1}{4} \cos (2 n+p) \theta+\frac{1}{4} \cos (2 n-p) \theta$

$$
\begin{aligned}
& +\frac{1}{4} \cos (2 n+2+p) \theta+\frac{1}{4} \cos (2 n+2-p) \theta \\
& -\frac{1}{2} \cos (2 n+1+p) \theta-\frac{1}{2} \cos (2 n+1-p) \theta \\
& -\frac{1}{2} \cos (1+p) \theta-\frac{3}{2} \cos (1-p) \theta .
\end{aligned}
$$

Denote $I(p, \theta)=\int \kappa(p, \theta) d \theta ;$ then

(A.4) $I(p, \theta)=L_{0}-L_{1}+L_{2 n}-L_{2 n+1}+L_{2 n+2}-M_{1}+M_{2 n}-M_{2 n+1}+M_{2 n+2}$

where

$$
\begin{aligned}
L_{m} & =\theta / q_{m} & & \text { if } p=m, \\
& =\sin (m-p) \theta / q_{m}(m-p) & & \text { otherwise, }
\end{aligned}
$$

and

$$
M_{m}=\sin (m+p) \theta / q_{m}(m+p)
$$

with $q_{0}=1, q_{1}=q_{2 n+1}=2, q_{2 n}=q_{2 n+2}=4$.

Therefore

$$
\begin{aligned}
a_{p} & =\frac{1}{2 \pi} \sum_{r=1}^{2 n+1}(-1)^{r-1}[I(p, \theta)]_{\theta=(r-1) \theta_{n}}^{\theta=r \theta} n \\
& =\frac{1}{2 \pi}[I(p, 2 \pi)-I(p, 0)\}+\frac{1}{\pi} \sum_{r=1}^{2 n}(-1)^{r-1} I\left(p, r \theta_{n}\right) .
\end{aligned}
$$

Cases of special interest occur when

$p=0,1, \ldots, n-1, n, n+1, n+2, \ldots, 2 n, 2 n+1$,

$$
2 n+2, \ldots, 3 n, 3 n+1,3 n+2,3 n+3, \ldots .
$$

For $p=0$,

$$
I(0, \theta)=\theta+\frac{\sin 2 n \theta}{4 n}+\frac{\sin (2 n+2) \theta}{(4 n+4)}-\frac{\sin (2 n+1) \theta}{(2 n+1)}-\sin \theta .
$$

Since for any $N$,

$$
\sum_{r=1}^{2 n}(-1)^{r-1} \sin N r \theta_{n}=\tan \left(N \frac{\theta_{n}}{2}\right) \text {, }
$$

and 


$$
\sum_{r=1}^{2 n}(-1)^{r-1}{ }_{r \theta_{n}}=-n \theta_{n},
$$

then substitution in equation (A.5) gives

$$
a_{0}=\frac{1}{2 n+1}-\frac{1}{\pi}\left\{1+\frac{1}{4 n(n+1)}\right\} \tan \theta_{n} \text {. }
$$

In a similar fashion we obtain the following values:

(A.7)

$$
\begin{aligned}
a_{1}=\frac{-1}{2(2 n+1)}+\frac{1}{\pi}\{(1+ & \left.\frac{1}{4 n(n+1)}\right) \tan \left(\frac{\theta^{\theta}}{2}\right)- \\
& \left.-\left(\frac{1}{4}+\frac{1}{(2 n-1)(2 n+3)}\right) \tan \theta_{n}\right\},
\end{aligned}
$$

(A.8) $a_{n-1}=\frac{1}{\pi}\left\{\left(-\frac{1}{2 n}-\frac{1}{4(n+1)}+\frac{1}{4(3 n+1)}\right) \cot \left(\frac{n}{4}\right)+\right.$

$$
\begin{aligned}
& +\left(\frac{1}{n-1}+\frac{1}{2(n+2)}-\frac{1}{6 n}\right) \cot \left(\frac{3 \theta}{4}\right) \\
& \left.-\left(\frac{1}{2(n-2)}+\frac{1}{4(n+3)}+\frac{1}{4(3 n-1)}\right) \cot \left(\frac{5 \theta}{4}\right)\right\},
\end{aligned}
$$

(A.9) $\quad a_{n}=\frac{1}{\pi}\left\{\left(\frac{5}{4 n}+\frac{1}{n+1}-\frac{1}{2(3 n+1)}-\frac{1}{4(3 n+2)}\right) \cot \left(\frac{n}{4}\right)\right.$

$$
\left.-\left(\frac{1}{2(n-1)}+\frac{1}{4(n+2)}-\frac{1}{12 n}\right) \cot \left(\frac{3 \theta}{4}\right)\right\} \text {. }
$$

(A.10) $a_{n+1}=\frac{1}{\pi}\left\{\left(-\frac{1}{n}+\frac{1}{4(3 n+1)}-\frac{5}{4(n+1)}+\frac{1}{2(3 n+2)}\right) \cot \left(\frac{n}{4}\right)\right.$

$$
\left.+\left(\frac{1}{4(n-1)}+\frac{1}{2(n+2)}-\frac{1}{12(n+1)}\right) \cot \left(\frac{3 \theta}{4}\right)\right\} \text {, }
$$

(A.11) $a_{n+2}=\frac{1}{\pi}\left\{\left(\frac{1}{4 n}+\frac{1}{2(n+1)}-\frac{1}{4(3 n+2)}\right) \cot \left(\frac{n}{4}\right)\right.$

$$
\begin{aligned}
& -\left(\frac{1}{2(n-1)}+\frac{1}{(n+2)}-\frac{1}{6(n+1)}\right) \cot \left(\frac{3 \theta n}{4}\right) \\
& \left.+\left(\frac{1}{4(n-2)}+\frac{1}{2(n+3)}-\frac{1}{4(3 n+4)}\right) \cot \left(\frac{5 \theta n}{4}\right)\right\},
\end{aligned}
$$


266

B.J. Noye

(A.12)

$$
\begin{aligned}
a_{2 n}=\left(\frac{1}{4}-\frac{n}{2(2 n+1)}\right)+ & \frac{1}{2 \pi}\left\{\left(-1-\frac{1}{n}+\frac{1}{(4 n+1)}\right) \tan \left(\frac{n}{2}\right)+\right. \\
+ & \left.\left(\frac{3}{4}-\frac{1}{8 n}+\frac{1}{(2 n-1)}\right) \tan \theta_{n}\right\},
\end{aligned}
$$

(A.13) $a_{2 n+1}=\left(-\frac{1}{2}+\frac{n}{2 n+1}\right)+\frac{1}{2 \pi}\left\{1+\frac{1}{2 n}-\frac{1}{2(n+1)}-\frac{1}{2(4 n+1)}\right.$

$$
\left.+\frac{1}{2(4 n+3)}\right\} \tan \left(\frac{\theta}{2}\right) \text {, }
$$

(A.14) $a_{2 n+2}=\left(\frac{3}{4}-\frac{n}{2(2 n+1)}\right)+\frac{1}{2 \pi}\left\{\left(-1+\frac{1}{(n+1)}-\frac{1}{(4 n+3)}\right) \tan \left(\frac{\theta^{\theta}}{2}\right)+\right.$

$$
\left.+\left\{\frac{1}{4}-\frac{1}{(2 n+3)}+\frac{1}{8(n+1)}\right) \tan \theta_{n}\right\} \text {, }
$$

(A.15)

$$
\begin{aligned}
a_{3 n}=\frac{1}{\pi}\left\{\left(\frac{1}{4 n}-\frac{1}{2(3 n+1)}+\right.\right. & \left.\frac{1}{4(5 n+2)}\right) \cot \left(\frac{\theta_{n}}{4}\right) \\
& -\left(\frac{1}{2(n-1)}-\frac{1}{3 n}+\frac{1}{2(5 n+1)}\right) \cot \left(\frac{3 \theta}{4}\right) \\
& \left.+\left(\frac{1}{4(n-2)}-\frac{1}{2(3 n-1)}+\frac{1}{20 n}\right) \cot \left(\frac{5 \theta n}{4}\right)\right\},
\end{aligned}
$$

(A.16)

$$
\begin{aligned}
a_{3 n+1}=\frac{1}{\pi}\left\{\left(-\frac{1}{2 n}-\frac{1}{4(n+1)}\right.\right. & +\frac{1}{(3 n+1)}+\frac{1}{2(3 n+2)}-\frac{1}{2(5 n+2)}- \\
& \left.-\frac{1}{4(5 n+3)}\right) \cot \left(\frac{n}{4}\right) \\
& \left.+\left(\frac{1}{4(n-1)}-\frac{1}{6 n}+\frac{1}{4(5 n+1)}\right) \cot \left(\frac{3 \theta n}{4}\right)\right\},
\end{aligned}
$$

$(\mathrm{A} .17)$

$$
\begin{aligned}
a_{3 n+2}=\frac{1}{\pi}\left\{\left(\frac{1}{4 n}+\frac{1}{2(n+1)}-\right.\right. & \frac{1}{2(3 n+1)}-\frac{1}{(3 n+2)}+\frac{1}{4(5 n+2)}+ \\
& \left.+\frac{1}{2(5 n+3)}\right) \cot \left(\frac{n}{4}\right) \\
& \left.-\left(\frac{1}{4(n+2)}-\frac{1}{6(n+1)}+\frac{1}{4(5 n+4)}\right) \cot \left(\frac{3 \theta_{n}}{4}\right)\right\},
\end{aligned}
$$

https://doi.org/10.1017/S0004972700045007 Published online by Cambridge University Press 
(A.18) $a_{3 n+3}=\frac{1}{\pi}\left\{\left(\frac{1}{4(n+1)}-\frac{1}{2(3 n+2)}+\frac{1}{4(5 n+3)}\right) \cot \left(\frac{n}{4}\right)\right.$

$$
\begin{aligned}
& +\left(\frac{1}{2(n+2)}-\frac{1}{3(n+1)}+\frac{1}{2(5 n+4)}\right) \cot \left(\frac{3 \theta_{n}}{4}\right) \\
& \left.-\left(\frac{1}{4(n+3)}-\frac{1}{2(3 n+4)}+\frac{1}{20(n+1)}\right) \cot \left(\frac{5 \theta}{4}\right)\right\} .
\end{aligned}
$$

\section{References}

[1] A. Brown, "On the differential equations for tide-well systems", BuZZ. Austral. Math. Soc. 5 (1971), 175-185.

[2] B.J. Noye, "The frequency response of a tide-well", Proc. 3rd Austral. Conf. on Hydraulics and Fluid Mechanics, Sydney, 1968, 65-71.

[3] B.J. Noye, "On a class of differential equations which model tide-well systems", Bull. Austral. Math. Soc. 3 (1970), 391-411.

[4] J.R. Rossiter and G.W. Lennon, "An intensive analysis of shallow water tides", Geophys. J.R. Astron. Soc. 16 (1968), 275-293.

[5] B.D. Zetler and R.A. Cummings, "A harmonic method for predicting shallow-water tides", J. Marine Res. 25 (1967), 103-114.

Department of Applied Mathematics,

University of Adelaide,

Adelaide,

South Australia. 